\title{
Nanopatterning Gold by Templated Solid State Dewetting on the Silica Warp and Weft of Diatoms
}

\author{
Jon Hiltz, ${ }^{1}$ Ahmadreza Hajiaboli, ${ }^{1}$ Gursimranbir Singh, ${ }^{1}$ R. Bruce Lennox, \\ Gregor F. Fussmann, ${ }^{2}$ Etienne Low-Decarie, ${ }^{3}$ and Mark P. Andrews ${ }^{1}$ \\ ${ }^{1}$ Department of Chemistry, McGill University, 801 Sherbrooke St. West, Montreal, QC, Canada H3A 0B8 \\ ${ }^{2}$ Department of Biology, McGill University, 1205 Docteur Penfield, Montreal, QC, Canada \\ ${ }^{3}$ School of Biological Sciences, University of Essex, Wivenhoe Park, Colchester CO4 3SQ, UK \\ Correspondence should be addressed to Mark P. Andrews; mark.andrews@mcgill.ca
}

Received 27 June 2016; Accepted 29 August 2016

Academic Editor: Jorge Pérez-Juste

Copyright (C) 2016 Jon Hiltz et al. This is an open access article distributed under the Creative Commons Attribution License, which permits unrestricted use, distribution, and reproduction in any medium, provided the original work is properly cited.

\begin{abstract}
The diatom, Nitzschia palea, exhibits complex silica shell (frustule) topography that resembles the warp and weft pattern of woven glass. The surface is perforated with a rhombic lattice of roughly oblong pores between periodically undulating transverse weft costae. Exfoliated frustules can be used to template gold nanoparticles by thermally induced dewetting of thin gold films. Acting as templates for the process, the frustules give rise to two coexisting hierarchies of particle sizes and patterned distributions of nanoparticles. By examining temperature dependent dewetting of 5, 10, and $15 \mathrm{~nm}$ Au films for various annealing times, we establish conditions for particle formation and patterning. The $5 \mathrm{~nm}$ film gives distributions of small particles randomly distributed over the surface and multiple particles at the rhombic lattice points in the pores. Thicker films yield larger faceted particles on the surface and particles that exhibit shapes that are roughly conformal with the shape of the pore container. The pores and costae are sources of curvature instabilities in the film that lead to mass transport of gold and selective accumulation in the weft valleys and pores. We suggest that, with respect to dewetting, the frustule comprises 2-dimensional sublattices of trapping sites. The pattern of dewetting is radically altered by interposing a self-assembled molecular adhesive of mercaptopropyltrimethoxysilane between the Au film overlayer and the frustule. By adjusting the interfacial energy in this manner, a fractal-like overlay of Au islands coexists with a periodic distribution of nanoparticles in the pores.
\end{abstract}

\section{Introduction}

Ordered arrays of metallic nanoparticles on dielectric substrates and nanoholes in metal films feature large field enhancements for effects like surface enhanced Raman scattering, solar cells, label-free biosensors, and focusing optics [1-5]. Methods for making ordered arrays are manifold. They include drilling with focused ion beams [6], use of patterned photoresists [7], and stamps [8]. Thin metal films deposited from the vapor phase onto surfaces have high surface to volume ratio and therefore can exhibit strong capillary forces, given the significant energy differences between the film and substrate. These forces can cause the film to break up into islands and even nanosized particles. For example, a gold film of $\mathrm{nm}$ thickness is unstable on a nonplanar silica surface, though the film is kinetically frozen. The total free energy associated with the various interfaces of the film can be reduced if the film breaks up (dewets). When perturbed by heat, atomic diffusion occurs and the Au film dewets to form small particles. Since dewetting can occur at temperatures well below the melting temperature, the process is a solid state transformation.

In general, it is difficult to control the distribution and size of particles produced by dewetting unless some sort of constraint is applied. Selective dewetting of thin metal films on patterned substrates is one method to produce ordered nanoscale particles. Specific design rules for dewetting to regulate 2-dimensional (2D) and even $3 \mathrm{D}$ distributions of particles would be an advance for applications of metal nanoparticle arrays in catalysis, photonics, solar energy, and 
magnetic storage. Dewetting on lithographically patterned surfaces of silicon and silica has yielded nanoscale metal particles distributed according to the template pattern [913]. In other cases, ripple patterns have been produced in silica by oblique incidence ion etching [14], and surface reconstructions on alumina have been used to template dewetting [15]. There have been two reports of the use of silica bowl templates to orchestrate dewetting of coinage metal films into tunable and well-defined arrays of nanoparticles $[16,17]$. The resultant arrays showed strong surface enhanced Raman activity and systematic changes in extinction spectra.

In the biosphere, a recurring process in the algal phylum Bacillariophyta, single-cell and colonial diatoms, is the making of amorphous silica shells (frustules) into extraordinary hierarchies of ornate patterns over micrometre to nanometre length scales. This is accomplished in some $10^{5}$ extant diatom species with a high degree of reproducibility. Interest in diatoms for bioinspired materials and devices has elevated them to the status of paradigm for nanoscience and nanotechnology [18]. To date frustules have been used for micromoulding parts, anchoring biomolecules, bioassay, and catalysis [18-25]. Fang and coworkers [26] showed that amines could direct assembly of gold nanoparticles on various species of diatoms. For example, the frustules of Coscinodiscus sp. have been used to make nanostructured metallic microshells by dissolving away the gold coated frustule from vapour deposited Au metal films [26]. We have reported [27] that the frustule of the pennate diatom Nitzschia palea (Figure 1) exhibits a strong frequency dependent optical response when decorated with $\mathrm{Au}$ nanoparticles and films that conform approximately to the periodicity of the holes in the frustule.

In this paper we report the first study devoted to dewetting of metal films on frustules. Thus we use a silica construct derived from Nature instead of the classic toolbox of microand nanofabrication to template dewetting. We show that the frustule of Nitzschia palea can be viewed as consisting of interpenetrating rhombic lattices of silica valleys and holes ("potential wells"). The lattices are created by a warp and weft texture of silica costae and pores that comprise the frustule. These features can be used to trap and pattern Au nanoparticles produced by dewetting thin Au films. Because the valleys have larger dimensions than the holes, they partition large particles by trapping them in the valleys, whereas small particles are trapped in the holes. This gives rise to two rhombic lattices of particles. Dewetting on the frustule is a template-driven process, meaning that template architecture and mechanical properties are expected to play roles in determining particle shape and spatial distribution. We show in this paper that combinations of film thickness, temperature, and time can produce Au particles and islands of different sizes and spatial distribution on the frustule.

The response of dewetting to a template like that of the $N$. palea frustule invites new questions and comparisons with dewetting on other patterns created in silicon and dielectrics. For example, since interfacial energies are important for dewetting we modified the frustule/Au interface by grafting with mercaptopropyltrimethoxysilane to create a somewhat compliant hybrid interface where the sulfur atom makes a

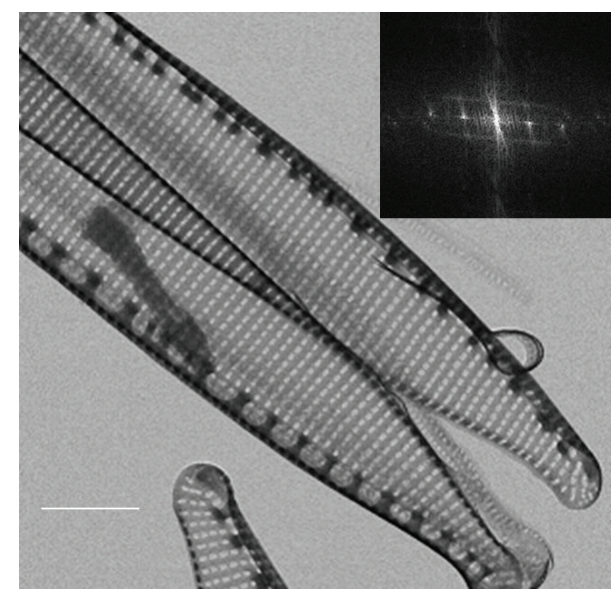

FIgURE 1: TEM image of exfoliated Nitzschia palea frustules. The upper valve half is the hypothecum, where the sternum of the raphe (slit) is visible as a thickened dark band. The bottom valve half epithecum shows the mating portion of the sternum. The raphe involves a thickened region of silica from which silica costae emanate to give a "warp and weft" structure to the basal silica plate (see AFM images, Figure 2). The nearly rectangular foramina (pore passageways) are distributed on a roughly rhombic lattice. The foramina are partially occluded by a perforated sieve plate (velum) that is not visible at this magnification. Cribellar pores in the sieve plate are arranged in roughly octagonal fashion and have diameters of about $20 \mathrm{~nm}$. Insert is the Fourier transform of the array of pores, confirming the rhombohedral arrangement. The scale bar is $2 \mu \mathrm{m}$.

covalent bond to gold. Compared with the pristine silica frustule surface, dewetting of gold films on the hybridized silica surface follows a different path to patterned Au.

We chose Nitzschia palea because this freshwater species is easily cultivated to give large quantities of silica frustules. It is a model organism, that is, for exploring effects of elevated $\mathrm{CO}_{2}$ on growth and competition in phytoplankton communities [28]. Certain metal ions in its nutrient environment can induce phenotypic abnormalities in the frustule [29]. This teratological response is interesting in itself because it suggests that the frustule can be modified without reengineering among the 70 genes that have been implicated in silica deposition [19].

\section{Experimental}

Nitzschia palea were obtained from the Canadian Phycological Culture Centre and kept as an axenic culture in $\mathrm{pH}$ 7 buffered nutrient medium. The cultures were continually shaken at $250 \mathrm{rpm}$ with a $3 \mathrm{~mm}$ rotation diameter to accelerate gas diffusion from the flask airspace to the culture medium. They were maintained in growth chambers under $100 \mu \mathrm{E}-\mathrm{m}^{-2} \mathrm{~s}^{-1}$ of continuous light at $25^{\circ} \mathrm{C}$. Organic material was removed from the diatoms by reaction with freshly prepared piranha solution. Following this treatment, the suspension was washed with distilled water and centrifuged repeatedly ( $4000 \mathrm{rpm}, 5$-minute cycles). Between each cycle, the resulting white pellet was washed with clean distilled $\mathrm{H}_{2} \mathrm{O}$, until the rinse was found to be $\mathrm{pH}$ neutral. The resulting 
suspension of cleaned frustules was stored in distilled water in a glass vial until needed.

Some frustule samples were functionalized with mercaptopropyltrimethoxysilane (MPTMS, Aldrich, 99\%) as follows: the suspension of diatoms was first centrifuged to obtain a pellet, which was resuspended in methanol $(\mathrm{MeOH}$, spectroscopic grade, Fischer, 99\%+, typically $40 \mathrm{~mL}$ ). To $40 \mathrm{~mL}$ solution of this $\mathrm{MeOH}$ suspension $100 \mu \mathrm{L}$ of MPTMS was added to make a $0.25 \%$ solution in a centrifuge tube. The resulting suspension was first stirred rapidly with a vortex mixer for 10 minutes and then shaken occasionally to encourage mixing over several hours. The suspension was left overnight to react. The suspension was subsequently centrifuged repeatedly and the pellet washed with clean $\mathrm{MeOH}$ in order to remove excess unreacted MPTMS. This typically required 3-5 cycles of centrifugation and rinsing. The chemically modified frustules were stored in clean $\mathrm{MeOH}$ until needed.

Fragments of piranha-cleaned $\mathrm{Si}$ wafer were used as substrates for annealing experiments. Frustules were spincoated or drop-cast onto the substrates from the stock $\mathrm{MeOH}$ solutions. Electron beam deposition of Au films was performed in a clean room facility using a Temescal bjd1800 evaporator operating at $9 \mathrm{kV}$ and $12 \mathrm{~A}$. The deposition rate was set to $2 \AA / \mathrm{s}$. The film thickness on the frustule was determined by an internal, calibrated microbalance sensor. Annealing was performed in air in a Lindberg horizontal tube furnace. Heating zones within the furnace were allowed to reach the desired annealing temperature prior to insertion of the samples. Once the allotted time had passed, the samples were moved to an unheated zone and allowed to cool slowly to prevent thermal shock upon removal of the sample.

Atomic Force Microscopy was conducted with a Bruker Nanoscope multimode 3 instrument, operating in contact mode, using a $100 \mu \mathrm{m}$ scanner. The samples were attached to magnetic pucks for analysis. SFR DNP-10 sharpened probes with nominal tip radii of curvatures of $20 \mathrm{~nm}$ and $2 \mathrm{~nm}$ and a force constant of $0.06-0.35 \mathrm{~N} / \mathrm{m}$ were used. Data were acquired, processed, and analyzed with Nanoscope Analysis software (version 1.20). Electron micrographs were obtained with a Hitachi S-4700 Field Emission Gun Scanning Electron Microscope (FESEM), operating at $2 \mathrm{kV}, 10 \mu \mathrm{A}$.

Control samples for Surface Enhanced Raman (SER) were prepared on glass microscope coverslips. Gold nanoparticles were first produced by dissolving $100 \mathrm{mg}$ of hydrogen tetrachloroaurate in $10 \mathrm{~mL}$ of water. This combination was added to a solution of $0.612 \mathrm{~g}$ of tetraoctylammonium bromide in $20 \mathrm{~mL}$ of toluene. The resulting two-phase mixture was stirred rapidly until all of the $\mathrm{AuCl}_{4}{ }^{-}$was transferred from the aqueous to the organic phase. Once phase transfer was complete, the reaction flask was placed in an ice bath where a solution of $105 \mathrm{mg}$ of sodium borohydride in $6 \mathrm{~mL}$ of water was added dropwise with vigorous stirring. The mixture was then removed from the ice and allowed to warm to room temperature under continuous stirring for several hours to complete the reaction. The resulting deep red suspension of toluene-soluble Au nanoparticles was then washed with MilliQ water and dried over sodium sulfate. The resulting composition was diluted to approximately $200 \mathrm{~mL}$ in toluene and stored until needed.
Glass microscope cover slips were then soaked in freshly prepared piranha solution $(3: 1$ concentrated sulfuric acid to $30 \%$ hydrogen peroxide) for 2 hours in a Teflon holder which was equipped with a magnetic stir bar. This process removes undesirable organic contaminants and makes the surface of the glass hydrophilic. The slides were then stirred in the same sample holder in MilliQ water for 5-minute intervals. The water was replaced at each interval, in order to remove excess acid. After thorough rinsing in MilliQ water, the slides were transferred in their sample holder into 95\% methanol and soaked for 30 minutes. The methanol was poured off and replaced. To this was added $0.25 \%$ by volume (approximately $50 \mu \mathrm{L}$ in $40 \mathrm{~mL}$ methanol) mercaptopropyltrimethoxysilane, which was stirred overnight in a covered beaker under ambient conditions. Following this reaction, remaining silane solution was decanted and the slides were rinsed alternatively with water and methanol. The surface modified slides were then stirred for several hours in suspensions of gold nanoparticles in order to chemically bind them to the surface.

Diatom substrates for SER spectra were prepared by dewetting $10 \mathrm{~nm}$ films of gold at $600^{\circ} \mathrm{C}$ for $2 \mathrm{~h}$. Diatoms were soaked in a dilute solution (approximately $50 \mathrm{mg}$ in $10 \mathrm{~mL}$ ) of 4-aminothiophenol in 95\% ethanol for 2 hours, removed, and rinsed with ethanol to remove excess thiophenol. The samples were then allowed to air-dry in a dust free environment prior to SER measurements. SER spectra were acquired with a JY LabRam HR confocal Raman microscope. The $633 \mathrm{~nm}$ line of a HeNe laser was used to excite the samples with a nominal power at the source of approximately $17 \mathrm{~mW}$. Neutral density filters were applied in order to reduce the incident laser fluence at the sample to prevent sample damage. The power incident on the sample surface through a $100 \mathrm{X}$ objective was measured between 0.1 and $2 \mathrm{~mW}$, depending on the filter employed. Spectra were acquired using 1800-line per $\mathrm{cm}$ grating and spectral integration times ranging from 5 to 60 seconds. Typically, 10 or 15 spectra were collected per sample, in the range of 400 to $1800 \mathrm{~cm}^{-1}$.

\section{Results and Discussion}

We first explore the structure of the frustule of Nitzschia palea by transmission, field emission scanning, and atomic force microscopy (TEM, FESEM, and AFM). This section is followed by an analysis of dewetting based on parameters of film thickness, temperature, and duration of annealing. The last section examines the impact of surface modification of the diatom with mercaptopropyltrimethoxysilane.

3.1. Nitzschia palea Silica Frustule Structural Features. Members of the diatom genus Nitzschia (family Bacillariaceae) are found in freshwater and marine environments. The species Nitzschia palea is easily cultivated from pure nutrient culture. Knowledge of the structural features of the silica shell, or frustule, is important to understand the pattern of dewetting of gold on the silica surface. The $N$. palea frustule comprises two halves that are visible in Figure 1 as exfoliated top (epithecum) and bottom (hypothecum) valve structures. When combined, the valves are demarcated by a 


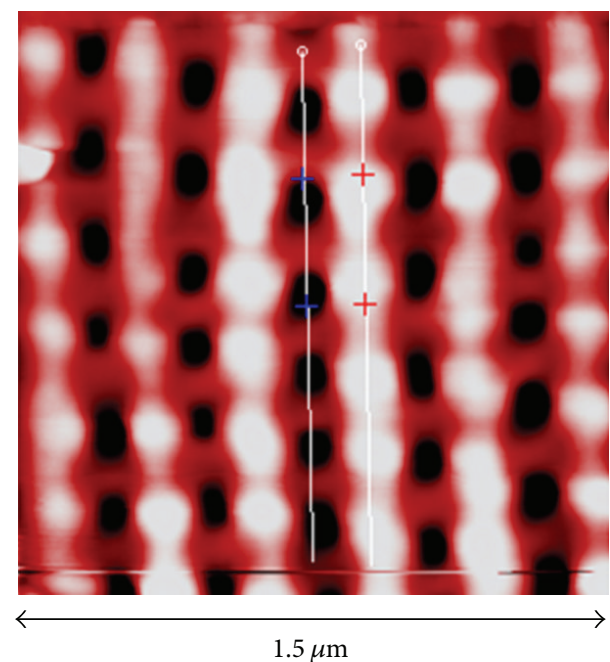

(a)

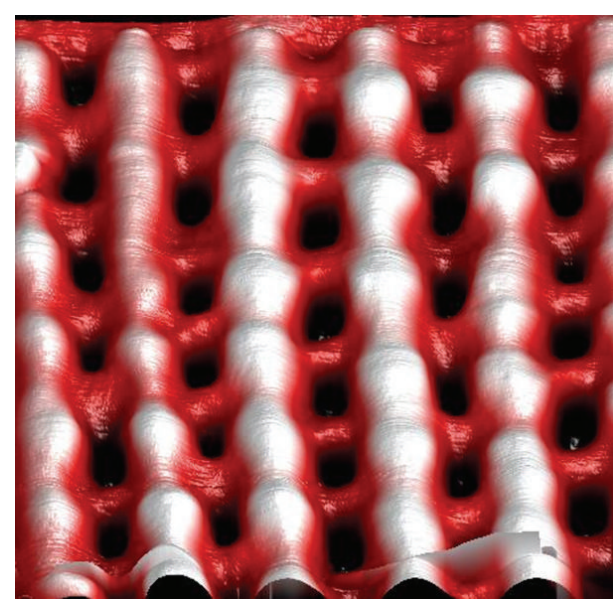

(b)

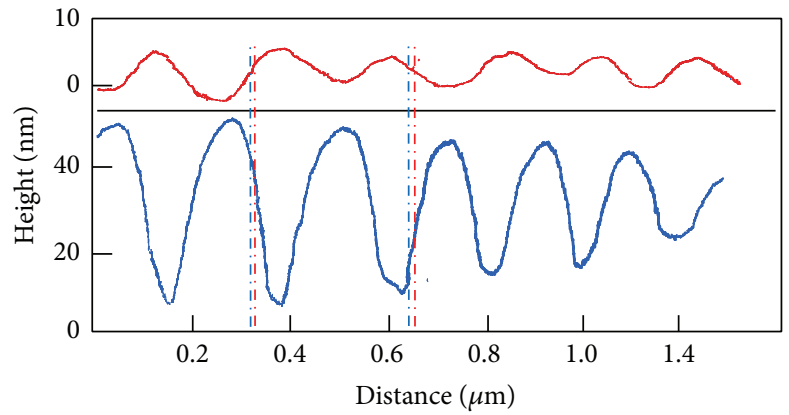

(c)

Figure 2: (a) AFM image of the top surface of an exfoliated Nitzschia palea frustule. The white regions (weft costae) run in the transverse direction. (b) Perspective of the image in (a). (c) Height profiles in the warp and weft regions of silica costae. The blue amplitude profile corresponds to the foramina (pores). The red profile corresponds to the peaks and valleys of the weft costae, where the peaks are observed to coincide with the minima of the openings.

longitudinal slit called the raphe, which is ostensibly used for motility. The raphe is defined by a silica sternum visible as the thickened dominant dark band in the TEM image of Figure 1. The sternum runs along the perimeter of the valve of the diatom. The pattern of holes near the raphesternum is often distorted and sparse. Lines of silica costae appear to emanate from the raphe-sternum (cf. AFM images in Figure 2). N. palea cells in our culture are typically $15-$ $20 \mu \mathrm{m}$ long and measure $3-4 \mu \mathrm{m}$ at the widest point, so the frustule is roughly shaped like a grain of rice as it tapers at both ends. When viewed from the top, the intact frustule (i.e., not inverted) is slightly convex in the middle along both axes, giving it an upside-down canoe-like shape. The basal silica layer of the frustule is perforated by a series of roughly rectangular passageways, or foramina (singular, foramen). These are not perfect rectangles, but they appear oblong in most cases. On average, the foramina measure $130 \mathrm{~nm} \times$ $60 \mathrm{~nm}$, with these dimensions reducing towards the frustule tip. The foramina are distributed in approximately periodic fashion on a rhombic lattice. Over the transverse axis, the foramina are spaced about $60 \mathrm{~nm}$ apart by silica. Along the longitudinal axis they are spaced by silica bands about $200 \mathrm{~nm}$ wide. Underlying each foramen is a partially occluding velum (not shown), which is a delicate latticework of silica comprising octagonally distributed cribral pores about $20 \mathrm{~nm}$ in diameter. The velum is also known as the sieve plate. The FFT image of a single frustule (insert of Figure 1) confirms that the arrangement of the large pores is rhombohedral. Overall then, the frustule manifests hierarchical periodicity over several length scales. AFM gives more insight into the frustule structure.

Figures 2(a) and 2(b) show an image of the top inside surface of a frustule valve half. The image was acquired in contact mode, which gave superior resolution over tapping mode. Neither tapping nor contact mode gave any evidence that the basal or costal structure is made up of nanograins of silica, like the 50-200 nm objects resolved in the frustule of Coscinodiscus granii [20, 21]. The surface clearly shows a great deal of three-dimensional texturing resembling the warp and weft pattern characteristic of woven glass mats. In the image, one can observe parallel ridges (the weft costae) along the transverse axis of the diatom. These are elevated with respect to the locations of the pores. The weft undulates in roughly periodic fashion with a peak to valley depth of 


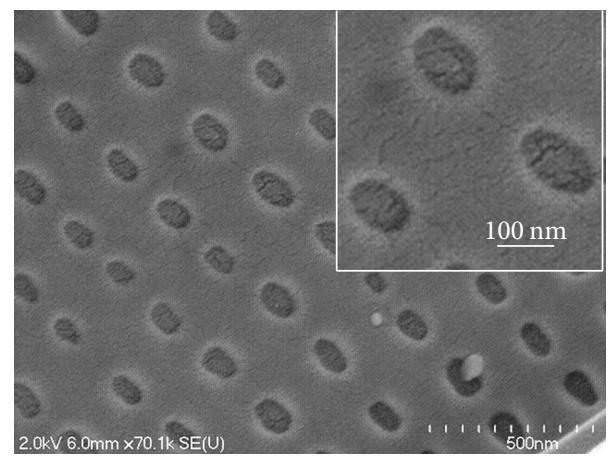

FIGURE 3: High resolution FESEM image of $10 \mathrm{~nm}$ gold film on the frustule surface prior to annealing. Dark patches represent the locations of the pores (magnification is $\times 70 \mathrm{k}$ ).

around $10 \mathrm{~nm}$, diminishing slightly towards the frustule edge. The peaks along the weft line up with the bottom of the pores. This is evident from the cross-section trace (blue trace) in Figure 2(c). The pores are between 40 and $50 \mathrm{~nm}$ deep. The depth is probably underestimated by AFM due to tip convolution. By AFM, the openings are roughly rectangular in shape with slightly rounded edges and average dimensions $\sim 130 \mathrm{~nm} \times \sim 60 \mathrm{~nm}$. These dimensions are very close to what are measured by TEM and SEM. In the valleys between the ridges, the rectangular pores are arranged with about a $200 \mathrm{~nm}$ period valley to valley. From a structural perspective, it is interesting to conjecture that the longitudinal and transverse silica costae may act as silica plate stiffeners in much the same way that structural I-beams do on perforated steel plates in marine structures like ship decks, lock gates, and floating docks. In both the marine organism and the human-engineered structure, the stiffening members resist axial compression due to sagging and hogging moments, particularly when the basal plate is perforated with holes like those in palea. In summary, the Nitzschia palea frustule exhibits complex 3D topography whose order can form the basis for nanoparticle size and positional self-correlations with long range regularity during metal film dewetting.

\subsection{Dewetting Experiments}

3.2.1. Gold Films on N. palea: Preliminaries. Gold films were deposited to a thickness of $10 \mathrm{~nm}$ by electron beam evaporation onto $N$. palea frustules on Si substrates. In Figure 3 we show a high magnification FESEM image of a $10 \mathrm{~nm}$ film before dewetting. The dark regions of contrast correspond to the underlying pore (foramina) structure. The film surface is grainy and shows stress relief defects in the form of spider and cohesive "mudflat" cracks, particularly within and around the edges of the pores.

The inset at higher magnification reveals that many cracks appear to radiate from the pore boundaries out into the interstitial regions. By AFM (Figure 4), the gold coated weft costae are clearly visible as lightly coloured vertical (transverse) bands in the image. These exhibit a grainy texture with root-mean-square roughness of $5 \mathrm{~nm}$, which falls within the typical range of $2-10 \mathrm{~nm}$ for evaporated gold films. The coating appears to be conformal, but close analysis of the surface amplitude profiles in the regions of the pores suggests that the pores are not filled. They are in fact coated with a membrane of gold. Pores that in uncoated N. palea are 40$50 \mathrm{~nm}$ deep appear to be only $3-5 \mathrm{~nm}$ deep in the coated sample. This is evident by comparing Figures 2 and 4 . The change in well-depth cannot be due to conformal filling of the pores down to the underlying silica sieve plate. Annealing the film at $400^{\circ} \mathrm{C}$ for 1 hour coarsens the grains and perceptibly opens the pores whose depths are observed to deepen to between 10 and $20 \mathrm{~nm}$. This is evident from Figures 4(a) and 4(b).

3.2.2. Gold Films on N. palea: Dewetting Experiments. We conducted experiments on 5, 10, and $15 \mathrm{~nm}$ Au films on $N$. palea. The primary objective was to see if dewetting could be controlled parametrically to deposit Au nanoparticles in the frustule pores and to see if hybrid structures of periodically sequestered nanoparticles might coexist with dewetted film overlayer morphologies. Accordingly, we set out to examine the impact of variations in film thickness, annealing temperature, and time on the outcome of templated dewetting on the silica frustule.

Films of $5 \mathrm{~nm}$ thickness were first annealed at the comparatively low temperature of $300^{\circ} \mathrm{C}$ for $60 \mathrm{~min}$. Within the frustule, a bird's-eye view shows the film in Figure 5(a) to be largely intact except near the tips and outer edges of the structure. There, discontinuities in the deposited film are most likely caused by shadowing during electron beam evaporation of the metal. Dewetting is known to initiate more easily from edge discontinuities like those created by shadowing [9]. The film deposited on the substrate dewets to give a fractal-like texture under these conditions. Dewetting into discrete particles is clearly incomplete on the frustule at this temperature and time scale, but it does show that the process is initiated well below the melting point of bulk gold $\left(1064.18^{\circ} \mathrm{C}\right)$. By contrast, when a $5 \mathrm{~nm}$ film is heated to $600^{\circ} \mathrm{C}$ for $10 \mathrm{~min}$, substantial dewetting is clearly evident. Figure 5(b) shows that $15-40 \mathrm{~nm}$ diameter particles are distributed randomly over the frustule basal silica costae in this instance. Particles on the order of $20-50 \mathrm{~nm}$ in diameter have also collected in the pore regions. The pores are occupied by single (larger) and in some cases multiple (smaller) particles. The "aerial" view inset of Figure 5(b) (1) gives a picture of two regimes of particle sizes: small particles captured in the periodic array of pores and small particles dispersed randomly on the frustule surface. On the substrate adjacent to the frustule (Figure 5(b) (2)), particles are randomly distributed and are larger in size than those located on the surface of the frustule, but comparable in size to those within the pores, which confers periodic correlations on the particles.

Gold films $5 \mathrm{~nm}, 10 \mathrm{~nm}$, and $15 \mathrm{~nm}$ thick were annealed at $600^{\circ} \mathrm{C}$ for increasing periods of time. Each time period is represented by a different sample so that the annealing time is not cumulative, but distinct. After $1 \mathrm{hr}$ annealing, the $5 \mathrm{~nm}$ film produced faceted particles with a mean diameter of about $50 \mathrm{~nm}$. These are randomly distributed on the surface. Similar sized single particles were deposited in the pores. This situation contrasts with that observed for $10 \mathrm{~nm}$ films. Figure 6 shows a progression in time for island and particle 

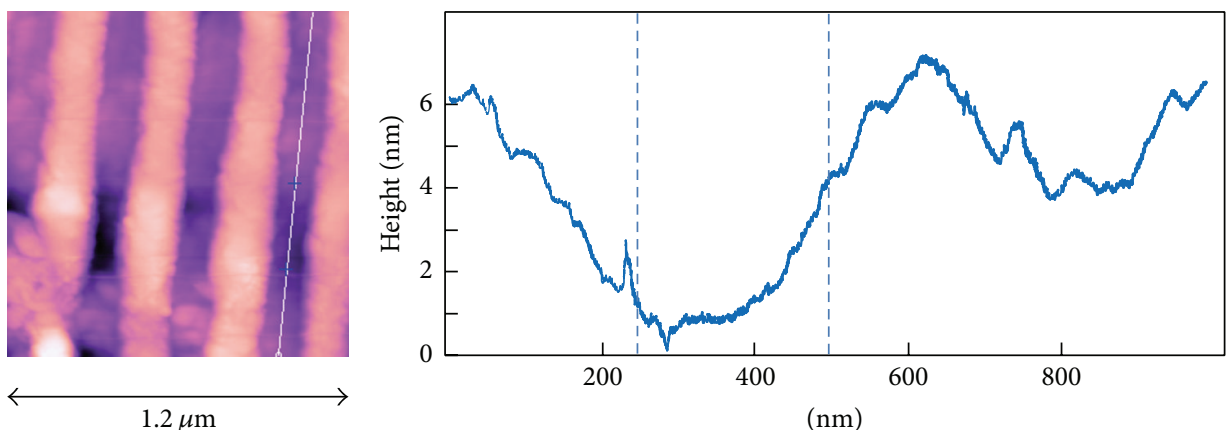

(a)
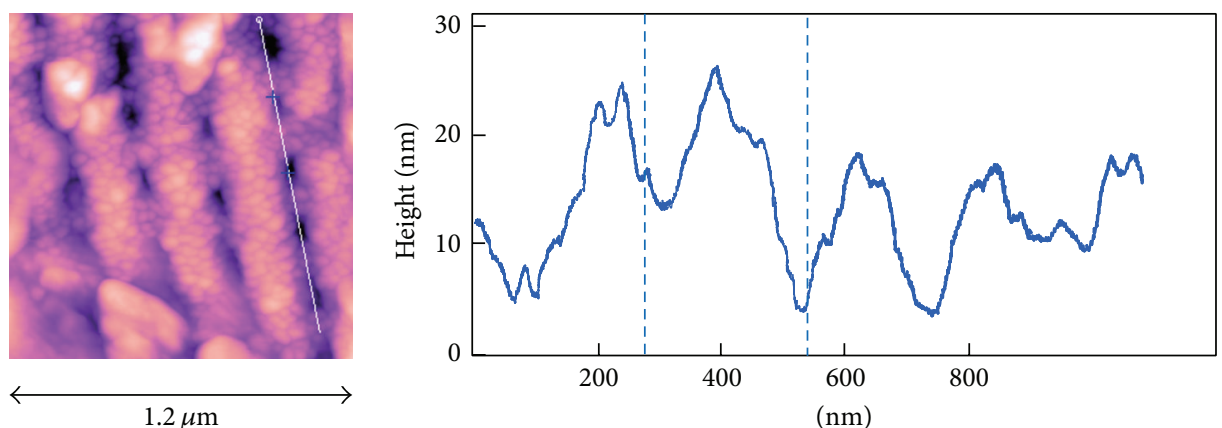

(b)

FIgURE 4: AFM images (left) and amplitude profiles (right) for a $10 \mathrm{~nm}$ Au film on N. palea. (a) Film as deposited. (b) Coarsening of surface texture observed after annealing $10 \mathrm{~nm}$ Au film in air at $400^{\circ} \mathrm{C}$ for $60 \mathrm{~min}$. The thick vertical bands are the Au coated weft costae transverse to the long axis of the diatom. Amplitude profiles were obtained in the valleys where the pores reside.

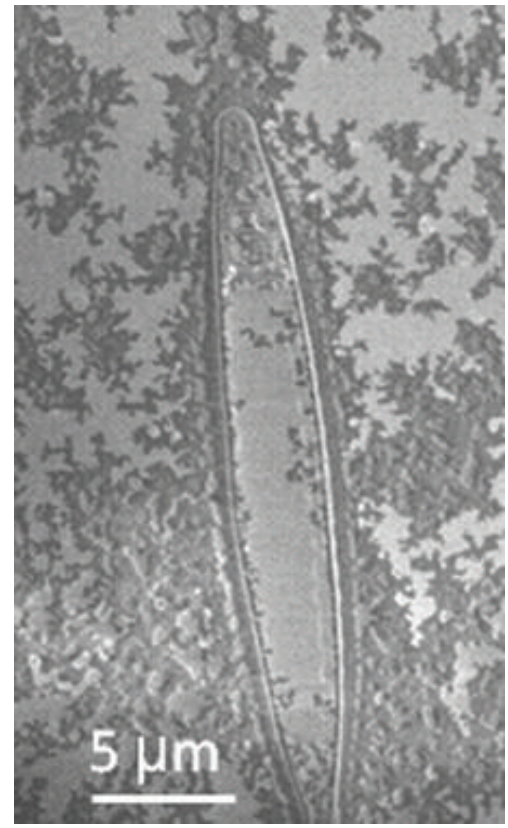

(a)
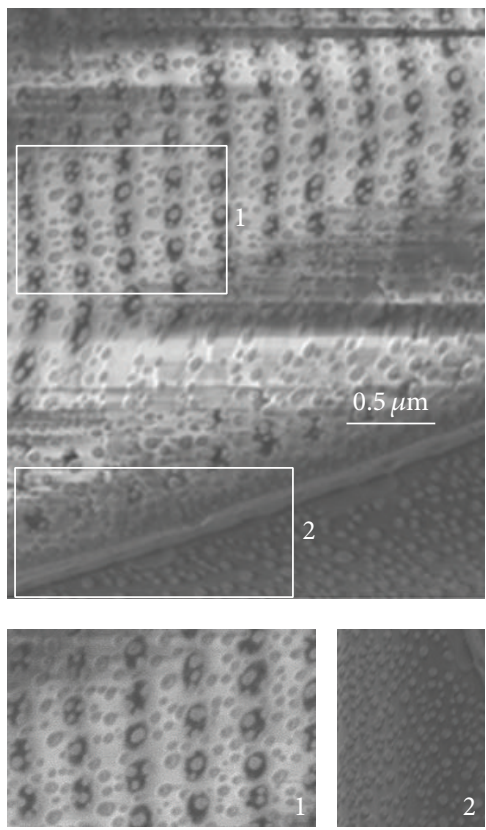

(b)

FIgURE 5: (a) FESEM image of $N$. palea coated with $5 \mathrm{~nm} \mathrm{Au}$ and annealed at $300^{\circ} \mathrm{C}$ for $60 \mathrm{~min}$. (b) shows the outcome of annealing a $5 \mathrm{~nm}$ $\mathrm{Au}$ film at $600^{\circ} \mathrm{C}$ for 10 minutes. Details of the particle distributions are compared at the bottom for N. palea in the magnification of region (1) and that of the adjacent silica-on-silicon substrate (2). In the latter case, the sternum associated with the raphe marks the boundary between the diatom and the substrate. Blurring of the image in (b) is caused by charging during FESEM image acquisition. 


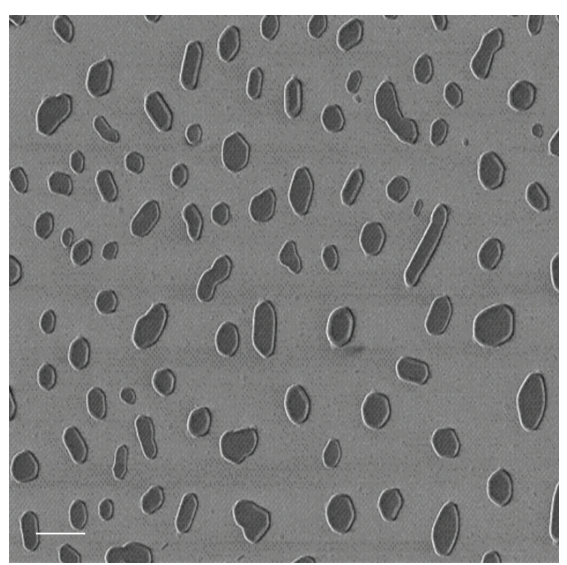

(a)

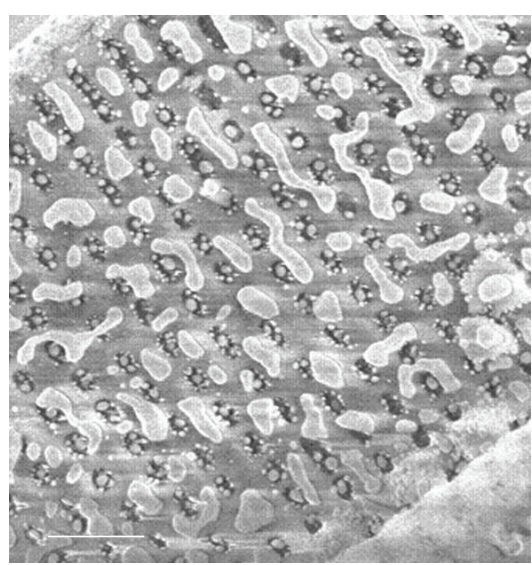

(b)

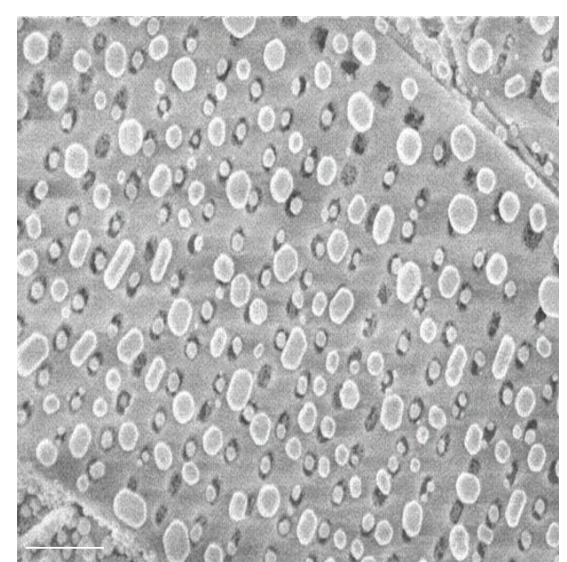

(c)

Figure 6: (a) FESEM image of Au particles after dewetting $10 \mathrm{~nm}$ Au film on silica-on-silicon substrate adjacent to $N$. palea at $600^{\circ} \mathrm{C}$ for $60 \mathrm{~min}$. (b) shows the outcome of annealing $N$. palea coated with $10 \mathrm{~nm}$ Au at $600^{\circ} \mathrm{C}$ for 60 minutes. (c) shows the outcome of annealing $N$. palea coated with $10 \mathrm{~nm} \mathrm{Au}$ at $600^{\circ} \mathrm{C}$ for 120 minutes. Scale bars $=0.5 \mu \mathrm{m}$.

formation on the substrate and on the frustule surface. After dewetting for $1 \mathrm{~h}$, particles on the substrate (Figure 6(a)) exhibit a range of shape morphologies and are substantially larger than those formed on the frustule after the same annealing period (Figure 6(b)). The substrate objects are broadly distributed in size, shape, and spacing. The frustule shows two regimes of objects after $1 \mathrm{~h}$ dewetting: small particles in the pores and islands on the costae.

This indicates that the time scale for formation of smaller particles in the pores is less than that for formation of individual large nanoparticles on the surface of the frustule. As discussed later, this can be explained by assuming that there are two regimes of Gibbs-Thompson excess chemical potential that control nanoparticle formation. After $2 \mathrm{~h}$ annealing (Figure 6(c)), small particles in the pores have coalesced into larger polyhedral particles, and the irregular island morphology on the surface has resolved into distinct nanoparticles. Results similar to Figure 6(c) were obtained for $15 \mathrm{~nm}$ thick films, although the surface particles were larger than those derived from the $10 \mathrm{~nm}$ film. The morphology observed in Figure 6(b) is reminiscent of Rayleigh-like instabilities [9-11] that precede breakup of structures into smaller particles. This system is the antecedent of the pattern obtained after $2 \mathrm{~h}$ annealing; see Figure 6(c). Note also in Figure 6(b) that the dewetted islands are elongated along the transverse costae. This orientational disposition is somewhat preserved when the islands break up into smaller polyhedral fragments on the top surface of the frustule. We argue that the spatial distribution of particles on two length scales (the pores on the one hand and the warp and weft on the other) originates by patterning on two interpenetrating quasi 2dimensional sublattices of the frustule. Each lattice offers its own periodic "potential wells" for capturing particles. This can be seen in the autocorrelations shown in Figure 7. These were obtained by converting images like those of Figure 6(c) into bit maps and thresholding for larger or smaller particles. Figure 7(a) shows autocorrelation for the large particles and it is clearly rhombohedral. The same is true in Figure 7(b) for the small particles that are located exclusively in the small pores. One lattice corresponds to minima in the weft costae valleys, hence, a lattice of potential wells for the large particles. The reason for locating the lattice for the large particles can be understood in reference to the AFM data of Figures 2(a) and 2(c): the minima in the amplitudes of the pore locations correspond approximately to maxima in the periodic undulations in the transverse weft, and vice versa.

We suggest that nanoparticle ordering is driven by templating the dewetting process through the excess chemical potential associated with different degrees of curvature on the 2D sublattices. Dewetting of Au films on the N. palea frustule is initiated by nucleation of defects/voids in the film. This is consistent with observations by others for templated dewetting [9-11]. Nucleation of voids (dry regions of silica) will be anisotropic for a film on a patterned surface, indicating anisotropy in material diffusion. Local thinning to produce dry regions (dewetting) is accomplished by mass transport to adjacent regions. Because the film is thin, its surface topography will replicate that of the frustule. This leads to local differences in chemical potential due to curvature, as expressed through the Gibbs-Thompson relation, $\Delta \mu=\kappa \gamma \Omega$, which relates the local excess chemical potential, $\Delta \mu$, to the local curvature, $\kappa$, surface energy, $\gamma$, and atomic volume, $\Omega$. For the undulating transverse weft costae there will be a positive excess chemical potential leading to anisotropic ("downhill") diffusion and an increase in thickness in the concave (valley) regions on one set of lattice points at the top surface. This will leave "dry" regions on the convex features of the costae. The formation of large particles with orientational distribution approaching biaxial on the silica surface is direct proof of this. Similarly, there must be a large excess chemical potential associated with the pore features, but here the driving force for the trapping potential for the smaller particles is much higher because of the greater curvature associated with the pore edges.

In addition to control of the length scales for pattern formation, it appears that the formation of ordered arrays, 


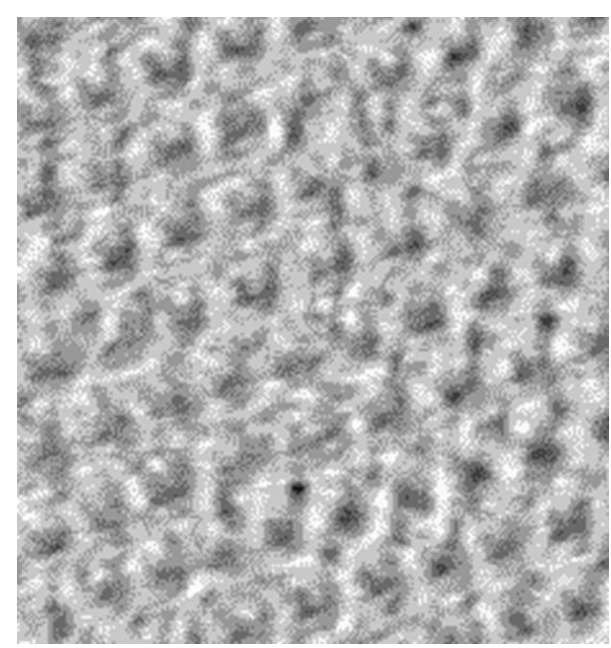

(a)

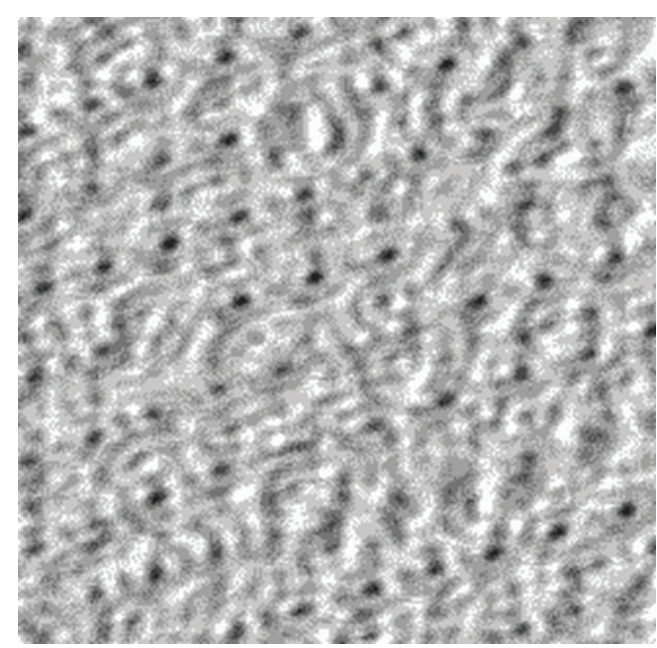

(b)

Figure 7: Autocorrelations for large particles (a) and small particles (b), for Au particles formed by dewetting $10 \mathrm{~nm}$ Au film on the N. palea frustule. Sample heated $2 \mathrm{~h}$ at $600^{\circ} \mathrm{C}$.

particularly on the weft structure, depends on the thickness of the film relative to the intrinsic geometric silica features. For nonoptimal film thickness (too thick or too thin) random particles are randomly distributed on the surface. This observation has been confirmed elsewhere in dewetting experiments on inverted pyramids in silicon templates [10].

There is a growing body of evidence that flexibility and defect fault tolerance can be built into metal films when they are deposited on compliant surfaces like polymers [30]. Since interfacial energies figure prominently in dewetting, we elected to adjust the surface energy on the frustule by decorating it with mercaptopropyltrimethoxysilane (MPTMS). This well-known "molecular adhesive" covalently bonds through siloxy moieties to the surface of the frustule through hydrolysis and polycondensation with adventitious surface water and silanol. Ideally, monolayer coverage can be achieved, although this is rarely the case in practice. The available thiol group will bind with deposited gold to produce a metal film whose mechanical properties are thought to be somewhat decoupled from the bare silica surface through the compliant molecular adhesive. Accordingly, MPTMS surface-modified frustules were coated with $10 \mathrm{~nm}$ gold films and dewetting experiments were carried out. Figure 8 reveals how the change in surface energy impacts the dewetting experiments. At $400^{\circ} \mathrm{C}$, there is little dewetting on the frustule after $4 \mathrm{~h}$ annealing (Figure 8(a)). By comparison, the $\mathrm{Au}$ film deposited on the substrate has broken up into islands with a fractal-like texture. This texture is not observed when gold films of the same thickness are dewetted at $450^{\circ} \mathrm{C}$ in the absence of the MPTMS interlayer. At $450^{\circ} \mathrm{C}$, the film on the frustule has dewetted into two strikingly different hierarchies of objects. There is a clear sublattice periodic array of rather uniformly sized Au nanoparticles in the pores (Figures $8(\mathrm{~b})-8(\mathrm{~d})$ ). This class of nanoparticles adopts dimensions close to those of the pores, so that not only are the particles periodically distributed but also shape anisotropy has been conferred on them due to the bounding container.
It is striking that a large component of the film adheres to the surface, giving areas of contiguous ramifying features with coarsened coastlines. Holes that appear in this overlayer correspond to the positions of the particles in the pores.

One explanation for the effect is that the MPTMS layer does not extend significantly over top of the pores, though MPTMS molecules may penetrate the cavity. In this view, the $\mathrm{Au}$ film overlayer would experience different dewetting behavior over the pore. Not only would there be a contribution to dewetting from curvature-driven instabilities, but also a lining of MPTMS in the pore would provide an additional chemical bond driving force to attract gold particles to the interior. Finally, heating the MPTMS-modified frustule to $600^{\circ} \mathrm{C}$ for $2 \mathrm{~h}$ recovers the pattern of nanoparticles (Figure 9) observed in the absence of the interfacial layer (compare Figure 6(c)). Overall then, chemical functionalization of the frustule surface markedly influences the dewetting morphology and the time scale for dewetting to reach two different regimes of particles. Chemical grafting therefore offers an additional variable to control metal nanoparticle formation. We are currently exploring this opportunity with different kinds of interfacial molecules.

While future work will examine the nanoplasmonic response of individual frustules in studies focused on understanding how these patterned metallodielectric hybrid structures interact with electromagnetic fields [27], we show in Figure 10 that a single diatom frustule can act as a surface enhanced Raman (SER) substrate to detect absorbed 4aminothiophenol. The diatom SER substrate was prepared by dewetting a $10 \mathrm{~nm}$ film of $\mathrm{Au}$ at $600^{\circ} \mathrm{C}$ for 60 minutes (compare Figure 6). As described in Section 2, a control was prepared by adsorbing 4-aminothiophenol onto 5-10 nm $\mathrm{Au}$ particles bound through the sulfur atom of mercaptopropyltrimethoxysilane to attach the nanoparticles to a silica glass coverslip. In Figure 10, the region between 1000 and $1200 \mathrm{~cm}^{-1}$ is dominated by the well-known spectroscopic signatures from the mixed CS stretching mode at $1075 \mathrm{~cm}^{-1}$ 


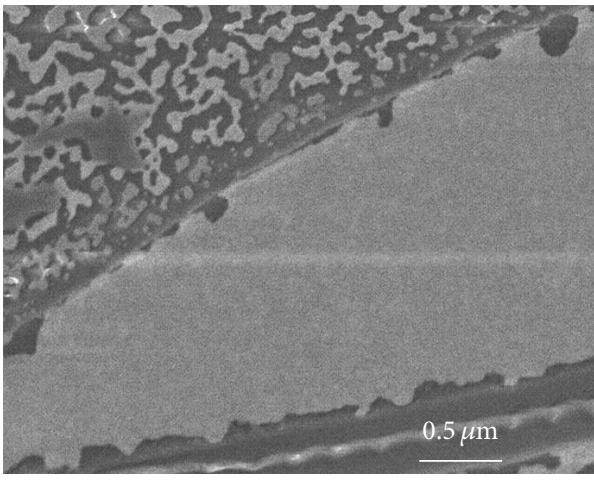

(a)

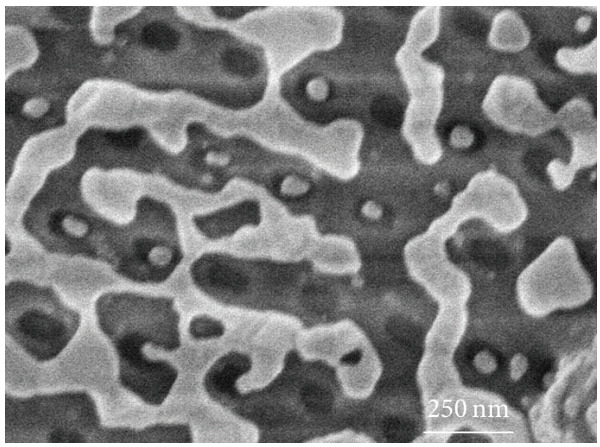

(c)

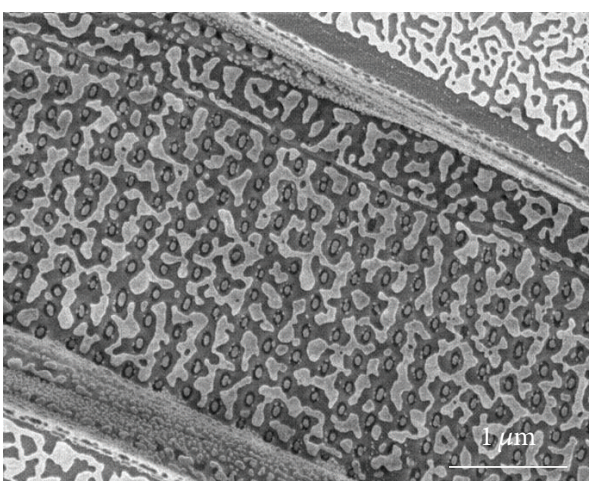

(b)

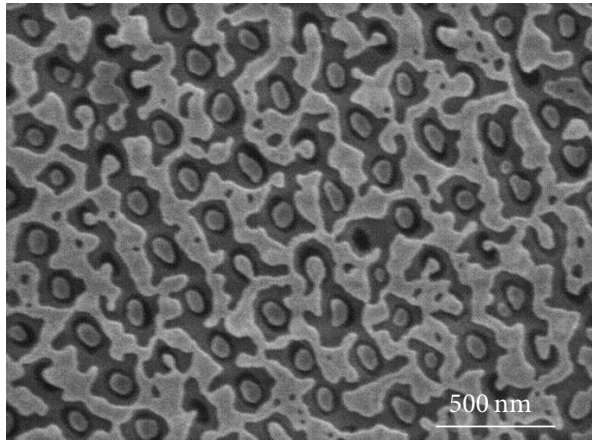

(d)

FIGURE 8: FESEM images. (a) Outcome of dewetting $10 \mathrm{~nm}$ Au film on Nitzschia palea frustule functionalized with MPTMS. (a) Film annealed at $400^{\circ} \mathrm{C}$ for $4 \mathrm{~h}$. (b-d) Separate sample annealed for 1 hour at $450^{\circ} \mathrm{C}$ and viewed at different magnifications. Compare with Figure $6(\mathrm{~b})$. Note in (a) and (b) the sharp boundary between the film on the substrate and the frustule sternum caused by shadowing during Au deposition.

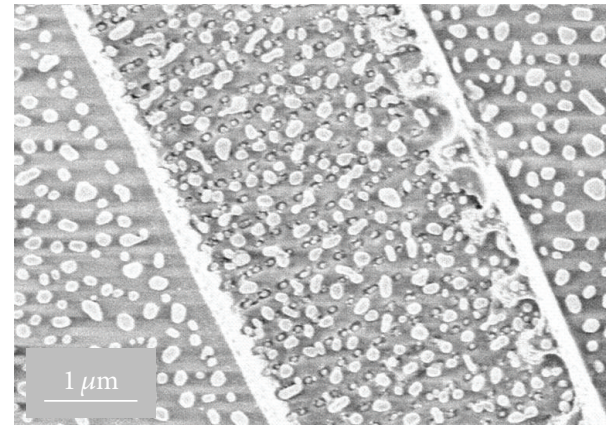

Figure 9: FESEM image. Outcome of dewetting $10 \mathrm{~nm}$ Au film on Nitzschia palea frustule functionalized with MPTMS. Film annealed at $600^{\circ} \mathrm{C}$ for $2 \mathrm{~h}$. Compare with Figure 6(c).

and the $\mathrm{CH}$ bending modes at $1141 \mathrm{~cm}^{-1}$ and $1180 \mathrm{~cm}^{-1}$ (the assignments are based on [31]). We observe that the bending mode at $1141 \mathrm{~cm}^{-1}$ is nearly equal in intensity to the CS stretching mode for SERS from the frustule and that it overlaps the weaker $a_{1}$ mode near $1180 \mathrm{~cm}^{-1}$. The SER spectrum from the thiophenol on the frustule differs from that obtained from the probe molecule bound to Au nanoparticles on the control substrate. This is evident by comparing the spectra in Figure 10(A) and (B). The spectrum (B) most closely resembles what is observed on roughened gold or gold nanoparticle substrates. The cause of the differences and the reason for the enhancement of the $9 b_{2}$ mode are unknown at this time; but they may originate in a wavelength dependent response of the periodic distribution of Au nanoparticles on the frustule surface. We have given evidence of such an effect elsewhere [27].

\section{Conclusions}

The frustule of Nitzschia palea is a highly textured topographical surface that is shown to lead to patterned dewetting of $\mathrm{Au}$ film overlayers. The dewetting process and pattern outcome depend on film thickness, temperature, and time. Under certain conditions, the thinnest films $(5 \mathrm{~nm})$ deposit the smallest particles in a two-level hierarchy: periodic patterning of single and multiple particles in the array of pores and random distributions of small particles on the frustule surface. Films on the order of $10 \mathrm{~nm}$ in thickness can be dewetted to give a second hierarchical class: periodic patterning of predominantly individual particles of similar size in the pores and large particles with a broad distribution of sizes but arranged with approximately biaxial order on the surface. Dewetting of films that are $15 \mathrm{~nm}$ thick gives the same hierarchical distribution as the $10 \mathrm{~nm}$ film, except that the surface particles are larger. AFM reveals that the surface of the frustule consists of silica costae that resemble the warp and weft of a weave. Analysis of the surface suggests that it offers an interpenetrating $2 \mathrm{D}$ rhombic sublattice for templating 


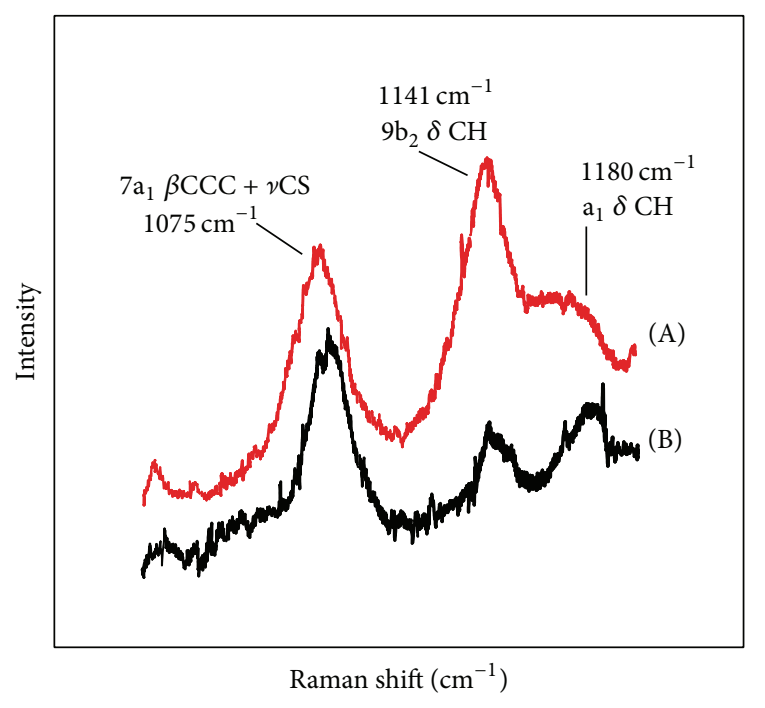

FIGURE 10: (A) SER spectrum from 4-aminothiophenol adsorbed on a single diatom prepared by dewetting a $10 \mathrm{~nm}$ Au film on Nitzschia palea frustule at $600^{\circ} \mathrm{C}$ for $2 \mathrm{~h}$. (B) SER spectrum from 4aminothiophenol adsorbed on 5-10 nm Au particles bound through mercaptopropyltrimethoxysilane (MPTMS) attached to a glass microscope coverslip.

particles. Large particles are trapped in a shallow periodic array of valleys built from the weft costae. Small particles are trapped in the deep potential of the periodic lattice of pores. This model is offered to explain the order over two size domains of particles. The dewetting phenomenon appears to be driven by excess chemical potential associated with curvature instabilities, voids, and defects. Chemical bonding of a compliant molecular layer to the surface of the frustule before thin film deposition markedly alters the dewetting process in time and in space. A change in interfacial energy for dewetting is implicated. Single frustules with gold nanoparticle prepared by dewetting show surface enhanced Raman scattering from adsorbed 4-aminothiophenol.

\section{Competing Interests}

The authors declare that there is no conflict of interests regarding the publication of this paper.

\section{Acknowledgments}

This research was made possible through the generous support of the Natural Sciences and Engineering Research Council (NSERC) of Canada. Additional support from the Centre for Self-Assembled Chemical Structures (CSACS) is gratefully acknowledged. The authors, moreover, thank Mohini Ramkaran for assistance with the AFM and Line Mongeon for TEM experiments.

\section{References}

[1] K. Kneipp, Y. Wang, H. Kneipp et al., "Single molecule detection using surface-enhanced Raman scattering (SERS)," Physical Review Letters, vol. 78, no. 9, pp. 1667-1670, 1997.
[2] A. Gopinath, S. V. Boriskina, B. M. Reinhard, and L. D. Negro, "Deterministic aperiodic arrays of metal nanoparticles for surface-enhanced Raman scattering (SERS)," Optics Express, vol. 17, no. 5, pp. 3741-3753, 2009.

[3] M. P. Jonsson, A. B. Dahlin, L. Feuz, S. Petronis, and F. Höök, "Locally functionalized short-range ordered nanoplasmonic pores for bioanalytical sensing," Analytical Chemistry, vol. 82, no. 5, pp. 2087-2094, 2010.

[4] K. R. Catchpole and A. Polman, "Plasmonic solar cells," Optics Express, vol. 16, no. 26, pp. 21793-21800, 2008.

[5] M. A. Seo, H. R. Park, S. M. Koo et al., "Terahertz field enhancement by a metallic nano slit operating beyond the skin-depth limit," Nature Photonics, vol. 3, pp. 152-156, 2009.

[6] A. G. Brolo, R. Gordon, B. Leathem, and K. L. Kavanagh, "Surface plasmon sensor based on the enhanced light transmission through arrays of nanoholes in gold films," Langmuir, vol. 20, no. 12, pp. 4813-4815, 2004.

[7] A. Javey and H. Dai, "Regular arrays of $2 \mathrm{~nm}$ metal nanoparticles for deterministic synthesis of nanomaterials," Journal of the American Chemical Society, vol. 127, no. 34, pp. 11942-11943, 2005.

[8] J. Henzie, J. Lee, M. H. Lee, W. Hasan, and T. W. Odom, "Nanofabrication of plasmonic structures," Annual Review of Physical Chemistry, vol. 60, pp. 147-165, 2009.

[9] D. Wang and P. J. Schaaf, "Two-dimensional nanoparticle arrays formed by dewetting of thin gold films deposited on prepatterned substrates," Journal of Materials Science: Materials in Electronics, vol. 22, no. 8, pp. 1067-1070, 2011.

[10] A. L. Giermann and C. V. Thompson, "Solid-state dewetting for ordered arrays of crystallographically oriented metal particles," Applied Physics Letters, vol. 86, no. 12, Article ID 121903, 2005.

[11] J. Ye and C. V. Thompson, "Templated solid-state dewetting to controllably produce complex patterns," Advanced Materials, vol. 23, no. 13, pp. 1567-1571, 2011.

[12] D. Kim, A. L. Giermann, and C. V. Thompson, "Solid-state dewetting of patterned thin films," Applied Physics Letters, vol. 95, Article ID 251903, 2009.

[13] C. M. Müller, F. C. F. Mornaghini, and R. Spolenak, "Ordered arrays of faceted gold nanoparticles obtained by dewetting and nanospherelithography," Nanotechnology, vol. 19, no. 48, Article ID 485306, 2008.

[14] J. Zhou, S. Facsko, M. Lu, and W. Möller, "Nanopatterning of Si surfaces by normal incident ion erosion: influence of iron incorporation on surface morphology evolution," Journal of Applied Physics, vol. 109, no. 10, Article ID 104315, 2011.

[15] J. Basu, C. B. Carter, R. Divakar, B. Mukherjee, and N. Ravishankar, "Nanopatterning by solid-state dewetting on reconstructed ceramic surfaces," Applied Physics Letters, vol. 94, no. 17, Article ID 171114, 2009.

[16] S. Yang, F. Xu, S. Ostendorp, G. Wilde, H. Zhao, and Y. Lei, "Template-confined dewetting process to surface nanopatterns: fabrication, structural tunability, and structure-related properties," Advanced Functional Materials, vol. 21, no. 13, pp. 24462455, 2011.

[17] S. Yang, B. Cao, L. Kong, and Z. Wang, "Template-directed dewetting of a gold membrane to fabricate highly SERS-active substrates," Journal of Materials Chemistry, vol. 21, no. 36, pp. 14031-14035, 2011.

[18] C. Jeffryes, J. Campbell, H. Li, J. Jiao, and G. Rorrer, “The potential of diatom nanobiotechnology for applications in solar cells, batteries, and electroluminescent devices," Energy and Environmental Science, vol. 4, no. 10, pp. 3930-3941, 2011. 
[19] R. Gordon, D. Losic, M. A. Tiffany, S. S. Nagy, and F. A. S. Sterrenburg, "The Glass Menagerie: diatoms for novel applications in nanotechnology," Trends in Biotechnology, vol. 27, no. 2, pp. 116-127, 2009.

[20] D. Losic, J. G. Mitchell, R. Lal, and N. H. Voelcker, "Rapid fabrication of micro- and nanoscale patterns by replica molding from diatom biosilica," Advanced Functional Materials, vol. 17, no. 14, pp. 2439-2446, 2007.

[21] D. Losic, J. G. Mitchell, and N. H. Voelcker, "Fabrication of gold nanostructures by templating from porous diatom frustules," New Journal of Chemistry, vol. 30, no. 6, pp. 908-914, 2006.

[22] N. L. Rosi, C. S. Thaxton, and C. A. Mirkin, "Control of nanoparticle assembly by using DNA-modified diatom templates," Angewandte Chemie, vol. 116, no. 41, pp. 5616-5619, 2004.

[23] J. Parkinson and R. Gordon, "Beyond micromachining: the potential of diatoms," Trends in Biotechnology, vol. 17, no. 5, pp. 190-196, 1999.

[24] A. R. Parker and H. E. Townley, "Biomimetics of photonic nanostructures," Nature Nanotechnology, vol. 2, no. 6, pp. 347-353, 2007.

[25] L. De Stefano, P. Maddalena, L. Moretti et al., "Nano-biosilica from marine diatoms: a brand new material for photonic applications," Superlattices and Microstructures, vol. 46, no. 1-2, pp. 84-89, 2009.

[26] Y. Fang, V. W. Chen, Y. Cai et al., "Biologically enabled syntheses of freestanding metallic structures possessing subwavelength pore arrays for extraordinary (surface plasmon-mediated) infrared transmission," Advanced Functional Materials, vol. 22, no. 12, pp. 2550-2559, 2012.

[27] M. P. Andrews, A. Hajiaboli, J. Hiltz, T. Gonzalez, G. Singh, and R. B. Lennox, "Nanoplasmonic photonic crystal diatoms and phytoliths," in Photonic and Phononic Properties of Engineered Nanostructures, vol. 79461 of Proceedings of SPIE, San Francisco, Calif, USA, January 2011.

[28] E. Low-Décarie, G. F. Fussmann, and G. Bell, "The effect of elevated $\mathrm{CO}_{2}$ on growth and competition in experimental phytoplankton communities," Global Change Biology, vol. 17, no. 8, pp. 2525-2535, 2011.

[29] E. Falasco, F. Bona, M. Ginepro, D. Hlúbiková, L. Hoffmann, and L. Ector, "Morphological abnormalities of diatom silica walls in relation to heavy metal contamination and artificial growth conditions," Water S A, vol. 35, no. 5, pp. 595-606, 2009.

[30] M. U. Manzoor, C. L. Tuinea-Bobe, F. McKavanagh et al., "Amorphous carbon interlayers for gold on elastomer stretchable conductors," Journal of Physics D: Applied Physics, vol. 44, no. 24, Article ID 245301, 2011.

[31] M. Osawa, N. Matsuda, K. Yoshii, and I. Uchida, "Charge transfer resonance Raman process in surface-enhanced Raman scattering from p-aminothiophenol adsorbed on silver: herzbergTeller contribution," Journal of Physical Chemistry, vol. 98, no. 48, pp. 12702-12707, 1994. 

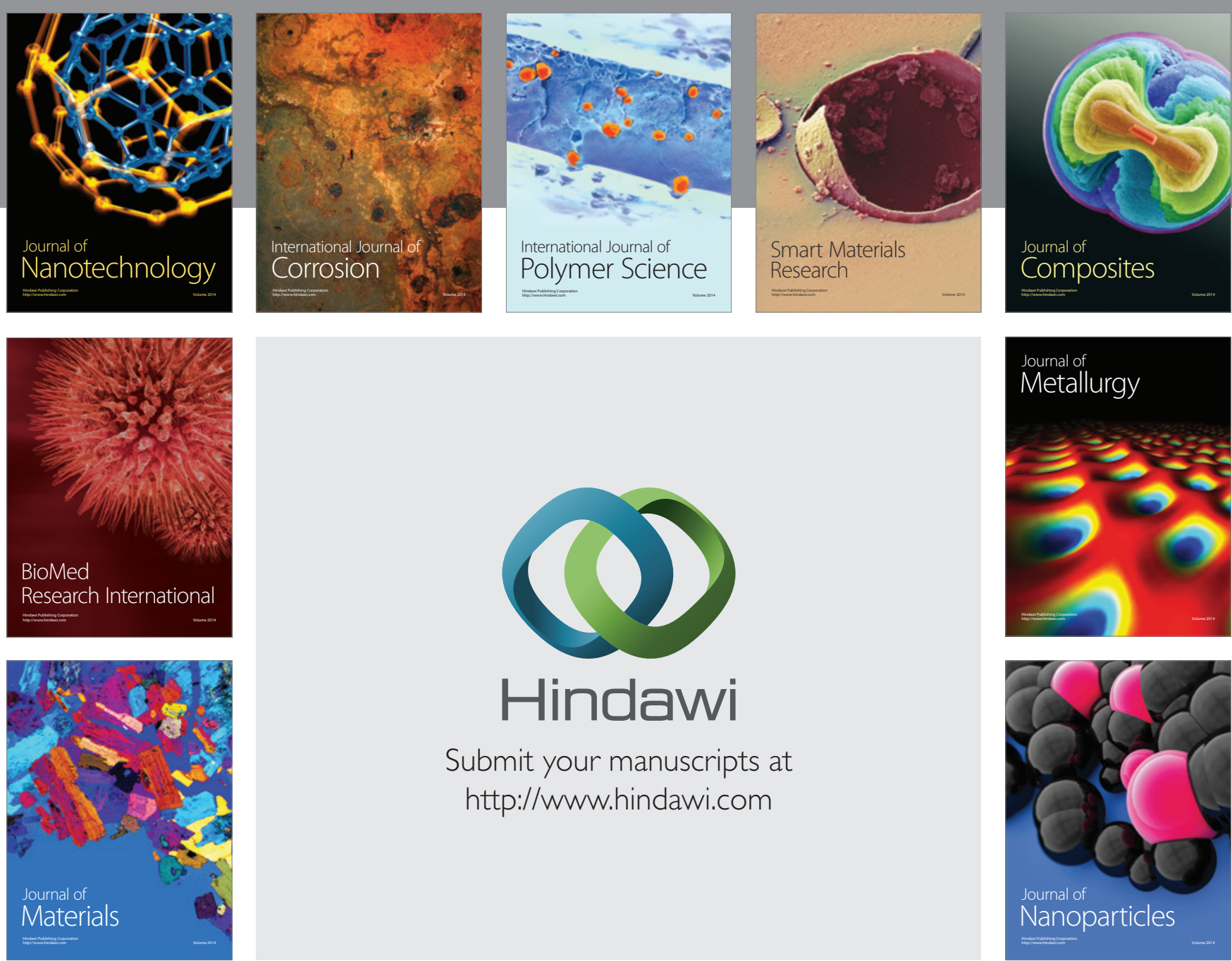

\section{Hindawi}

Submit your manuscripts at

http://www.hindawi.com

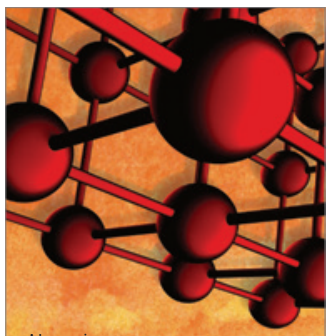

Materials Science and Engineering
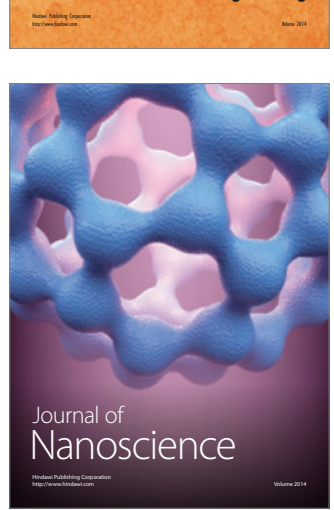
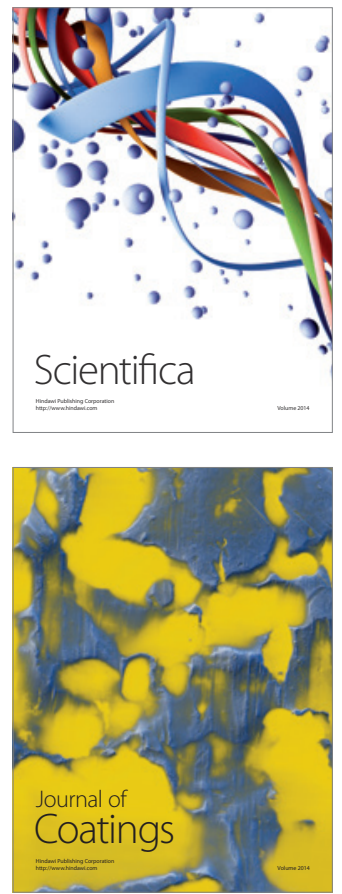
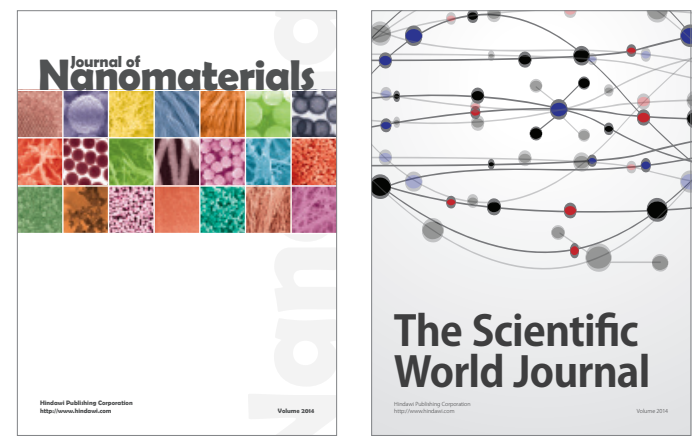

The Scientific World Journal
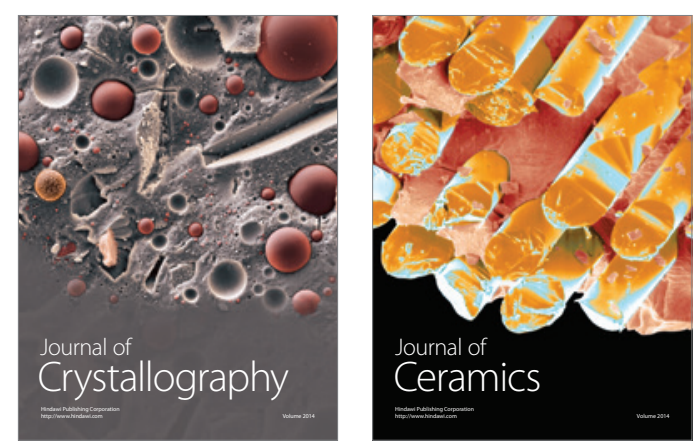
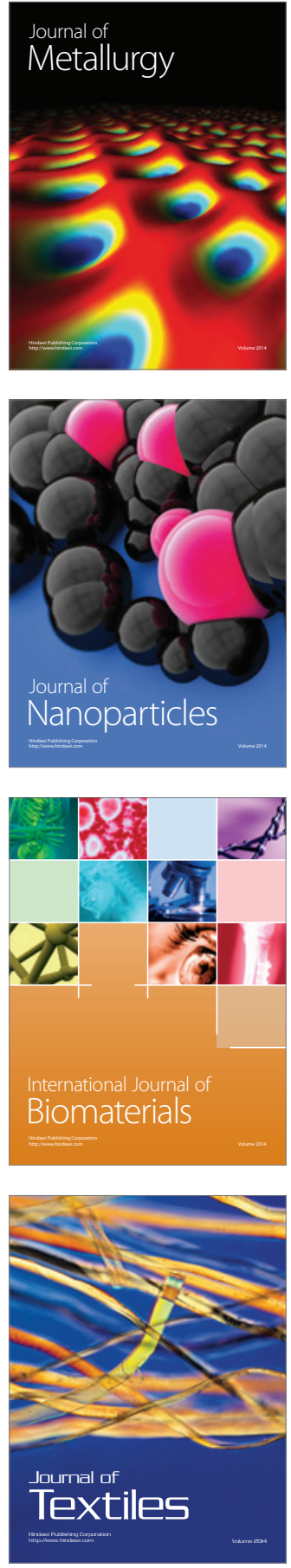